\title{
Automatically tracking feeding behavior in populations of foraging worms
}

\author{
Elsa Bonnard ${ }^{1 *}$, Jun Liu ${ }^{1 *}$, Nicolina Zjacic ${ }^{1,2}$, Luis Alvarez ${ }^{1} \&$ Monika Scholz ${ }^{1 \$}$ \\ 1 Max Planck Research Group Neural Information Flow, Max Planck Institute for Neurobiology of Behavior - \\ caesar, Bonn, Germany \\ 2 Institute of Medical Genetics, University of Zurich, Zurich, Switzerland \\ * These authors contributed equally to this work \\ \$To whom correspondence may be addressed: monika.scholz@mpinb.mpg.de
}

\begin{abstract}
C. elegans feeds on bacteria and other small microorganisms which it ingests using its pharynx, a neuromuscular pump. Currently, measuring feeding behavior requires tracking a single animal, indirectly estimating food intake from population-level metrics, or using restrained animals. Therefore, to enable large throughput feeding measurements of unrestrained, crawling worms on agarose plates, we developed an imaging protocol and a complementary image analysis tool called PharaGlow. We image up to 50 freely moving worms simultaneously and extract locomotion and feeding behaviors. Our tool reliably detects pharyngeal pumping in adult worms with a maximum deviation of $5 \%$ in the number of pumps compared to an expert annotator. We demonstrate the tool's robustness and highthroughput capabilities by measuring feeding in different use-case scenarios. This includes tracing pharyngeal dynamics during development, revealing their highly conserved nature throughout all life cycle stages. We also observed pumping after food deprivation, corroborating previous studies in which starvation time strongly influences pumping. Finally, we further validated our behavioral tracker by exploring two previously characterized pumping defective mutants: unc-31 and eat-18. Remarkably, our analysis of eat-18 mutants identified unreported defects in pumping and overall locomotion regulation, highlighting the potential of this toolkit. Pharaglow therefore enables the observation and analysis of the temporal dynamics of food intake with high-throughput and precision in a user-friendly system.
\end{abstract}

\section{Introduction}

Animals must forage for food to maintain their energy levels, grow and provide for their young. An optimal foraging strategy depends on a variety of environmental factors, such as the food distribution, predation risk, and competition ${ }^{1-4}$. Similarly, internal states, such as satiety or specific nutritional needs vary over time and modulate foraging behaviors ${ }^{5,6}$. Due to advances in animal tracking, locomotion towards food is well understood ${ }^{7-9}$, however, how feeding is coordinated with locomotion during foraging is less clear ${ }^{10}$. A key difficulty in studying food intake in foraging animals lies in the scarcity of tools available to perform quantitative and time-resolved measurements of feeding ${ }^{11}$. Due to its size, transparency and 
suitability for behavioral tracking, the roundworm $C$. elegans is an ideal model to advance our understanding of the factors influencing food intake and decision-making during foraging ${ }^{12,13}$.

C. elegans feeds on bacteria and other small microorganisms by drawing in a suspension of food particles from the environment. The food is ingested and separated from the liquid by the pumping action of its powerful pharyngeal muscles ${ }^{14-16}$. Transport proceeds with occasional peristaltic contractions that move food further toward the intestine where a hard cuticular structure, the grinder, crushes the bacteria before they are pushed into the intestine 17. Of these muscular motions, pumping is the most frequent contraction that is also the limiting step for food intake ${ }^{14-16}$. This process occurs up to 300 times per minute when food is abundant ${ }^{18-20}$. Feeding rates are altered in response to the type, concentration, size, and familiarity of the surrounding bacteria ${ }^{15,19-21}$. The behavioral and metabolic context, such as hunger, satiety, and mating drive also influence the rate of food intake ${ }^{22-25}$. Feeding behavior is thus regulated at different time scales ranging from immediate neuro-muscular activity ${ }^{26-29}$, to the intermediate scales of food choice and foraging ${ }^{12,13,30}$, to longer-term life history traits and behavioral state changes of the animal ${ }^{22,31}$.

Because of the transparent body of $C$. elegans, the pharynx can be directly observed through light microscopy, which in principle enables simultaneous detection of food particles (bacteria), muscular motion, and locomotion ${ }^{16}$. However, these experiments are often performed in immobilized animals, which can introduce artifacts in the observed behavior, as the activity of the body wall muscles feedbacks to the pharynx via parallel synaptic and hormonal routes ${ }^{32}$. While desirable, imaging feeding in moving animals, especially in large populations, is challenging due to the disparate time- and length scales of the motions involved. While worms move over centimeters within minutes ${ }^{33,34}$, the observable pharyngeal contractions are over $\mu \mathrm{m}$ within $\mathrm{ms}^{16}$, making large scale foraging experiments technically challenging.

Existing techniques to measure feeding fall broadly into two categories. The first focuses on indirect measures of population food intake, and the second detects each pumping contraction, albeit in a single worm at a time (Table 1). Indirect food intake measures rely either on labeling the food intake of the worm, for example using bioluminescent bacteria ${ }^{35}$, fluorescent bacteria ${ }^{24,36}$ or fluorescent beads ${ }^{16,37}$, or by measuring the remaining food concentration over time in large liquid cultures of worms ${ }^{38}$. Resolving single pump 
information can be achieved by combining brightfield microscopy with live worm tracking to remove center of mass motion and enable imaging of the grinder ${ }^{30,31,39}$, or alternatively by constraining animals in microfluidics. In tracking and constrained configurations, one can read out pumps by directly following the grinder motion in the pharynx ${ }^{19,20}$. A complementary technique relies on recording electropharyngeograms that detect the signature of muscular contractions without requiring a tracking microscope ${ }^{40}$. While some of the techniques that constrain animals allow simultaneous detection of a few animals, what is lacking is an approach that allows time-resolved pumping detection in populations of moving animals.

We wanted to fill the gap to allow imaging of feeding at high-throughput with single-pump temporal resolution in unrestrained animals, while using only optical setups already available in most $C$. elegans laboratories. Our method is based on epi-fluorescence microscopy of the pharyngeal muscle with a cost effective, large chip camera that enables imaging of many worms as they explore freely on an agarose plate. We determined that the method is relatively insensitive to the optical instrument used, and does not require high-end or custom optics. The accompanying analysis software (PharaGlow) is written in Python and can be accessed using the beginner friendly semi-graphical jupyter notebooks. PharaGlow is available under a permissive open source license. We demonstrate the usability and throughput of the method for multiple use cases, including those not previously possible in restrained animals, such as repeated imaging of a population of developing animals.

\section{RESULTS}

\section{Detection of pumping rates in crawling animals}

To enable automated, high-throughput detection of pumping in animals crawling on culture plates, we combined epi-fluorescence microscopy with a large area scan camera (Fig. 1A). Typically, pumping is detected by manual or automated counting using high magnification to resolve the motion of the grinder in the terminal bulb. We made use of animals expressing a fluorescent protein in the pharyngeal muscle, which allowed us to use a lower magnification compared to brightfield imaging of the grinder. For this, we use animals expressing YFP under the pharyngeal myosin promoter myo-2p (gnals1 [myo-2p::yfp]), which is expressed in the pharyngeal muscles, except pm1 and pm2 ${ }^{41,42}$.

By using a low magnification of $1 x$, we could image a field of view of 7 by $5 \mathrm{~mm}$, corresponding to multiple body lengths of the worms (Fig. 1A). We simultaneously imaged 
tens of animals (typically 30-50) as they crawled and analyzed their behavior off-line using our custom analysis software (Fig. 1B). The analysis pipeline combines a particle-tracking workflow with custom shape-segmentation of the fluorescent pharynx (Fig. 1C). After detecting and tracking the pharynges in the field of view, the contour and centerlines are fitted. The centerline and width are used to virtually straighten the animal (Fig. 1D). It then extracts a pump metric from the straightened images based on the standard deviation of the fluorescence along the dorsal-ventral axis (DV-axis) of the animal, which reflects pumping events (Fig. 1E, F). By averaging images during these putative detected pumping events, we determined that this metric is sensitive to the opening of the pharyngeal lumen and contraction of the terminal bulb and thus indeed corresponds to pumping events (Fig. 1G).

The low magnification ( $1 \mathrm{x}$ ) we use to image the animals allows us to increase the number of observed animals, but could compromise pumping detection. To determine how accurate our software detects pumping in these imaging conditions, we compared the results of our automated method and a manual annotator. Since manual annotation of pumping rates is still widely used, but practiced at higher magnifications, we first imaged worms at an effective magnification of $2 x$ (resolution: $1.17 \mu \mathrm{m} / \mathrm{px}$ ). A blinded human expert counted pumps in these videos. We then ran the automated analysis on the same video, but downscaled to $1 \mathrm{x}$ resolution. We found that pharaglow was able to accurately detect pumping in these videos (Fig. 2A), and consistently chose the same phase of the pharyngeal contraction. In contrast, the human annotator is less accurate in determining pump timing, which leads to spurious fluctuations when calculating the instantaneous pumping rate from the expert annotator's data (Fig. 2A, B). While the human annotator used the opening of the terminal bulb to define a pumping event, pharaglow detects the event at the maximal opening of the pharyngeal lumen (Fig. 2C). Both methods result in a comparable mean pumping rate for the animals counted (Fig. 2D, E), with a deviation between the human and automated results of less than $5 \%$ (Fig. 2F). Pharaglow is therefore able to automatically and reliably detect pumping in low-resolution, large field-of-view images. 


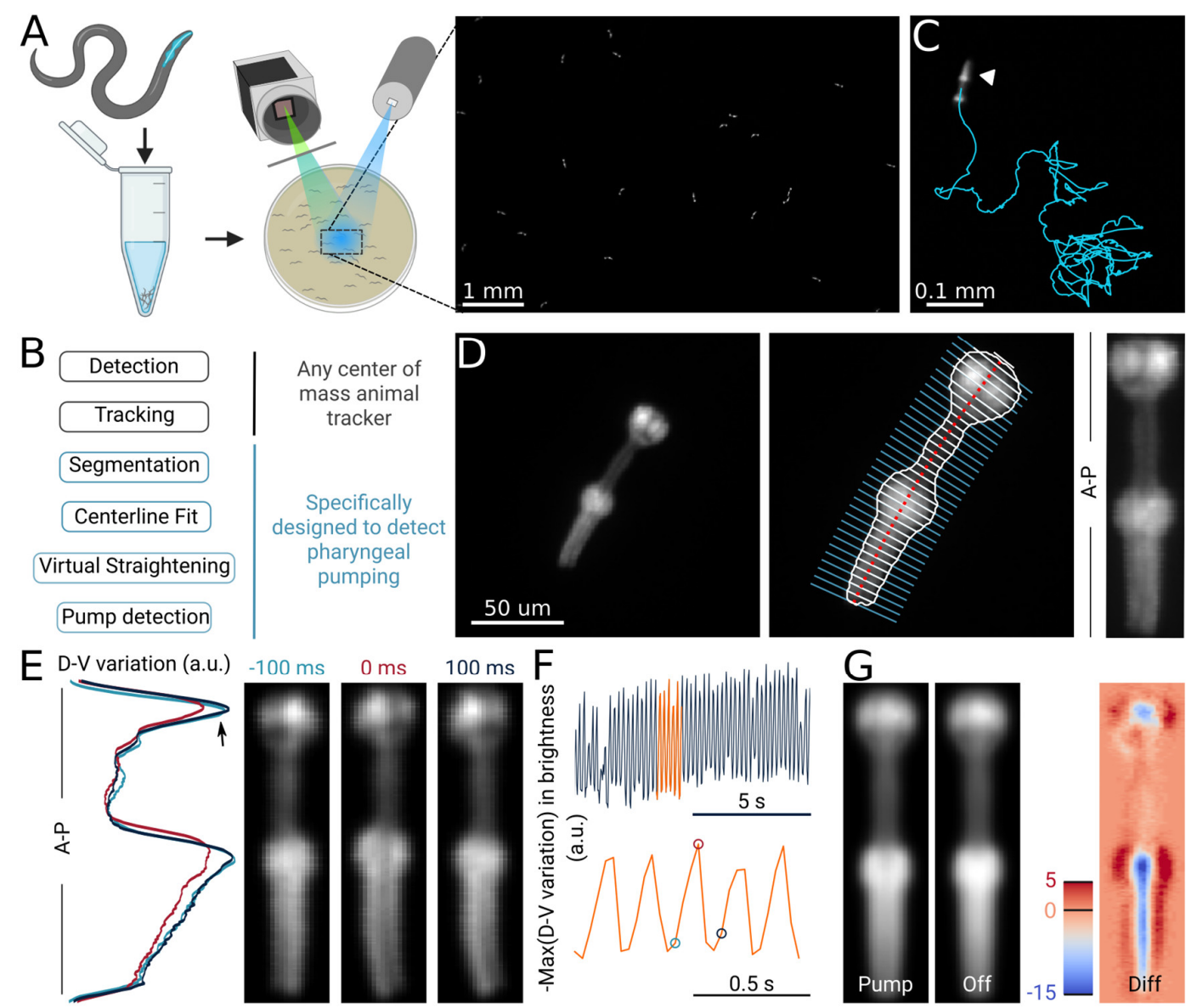

Figure 1. High-throughput optical detection of pharyngeal pumping in moving worms

(A) Hundreds of animals expressing myo-2p::YFP are washed in M9 and pipetted onto the assay plate before imaging with an epifluorescence microscope at $1 \mathrm{x}$ magnification resulting in a full field of view of 7 by $5 \mathrm{~mm}$. (B) Workflow of using the pharaglow image analysis pipeline. Animal center of mass tracking can be substituted with any available tracker, but subsequent steps are specific to tracking feeding. (C) Representative trajectory of an animal after tracking. (D) Processing steps followed for detection of pharyngeal pumping. Example of a fluorescent image (left; $2 x$ magnification). Segmentation of pharyngeal contour, centerline, and widths (middle) calculated for virtual straightening along the anterior-posterior axis (A.P.) and the resulting straightened animal (right). (E) Three straightened frames of an animal before, during, and after a pump and their dorso-ventral variation in brightness along the A.P. axis. (F) The metric that is used to detect pumping events. Bottom, a portion of the top trace (orange). Highlighted time points correspond to the images in (E). (G) Average of all images during a detected pump ('Pump') and for all remaining timepoints ('Off'). The difference image ('Diff') shows that pumps are characterized by the opening of the lumen and terminal bulb. Colorbar indicates brightness difference (a.u.) 


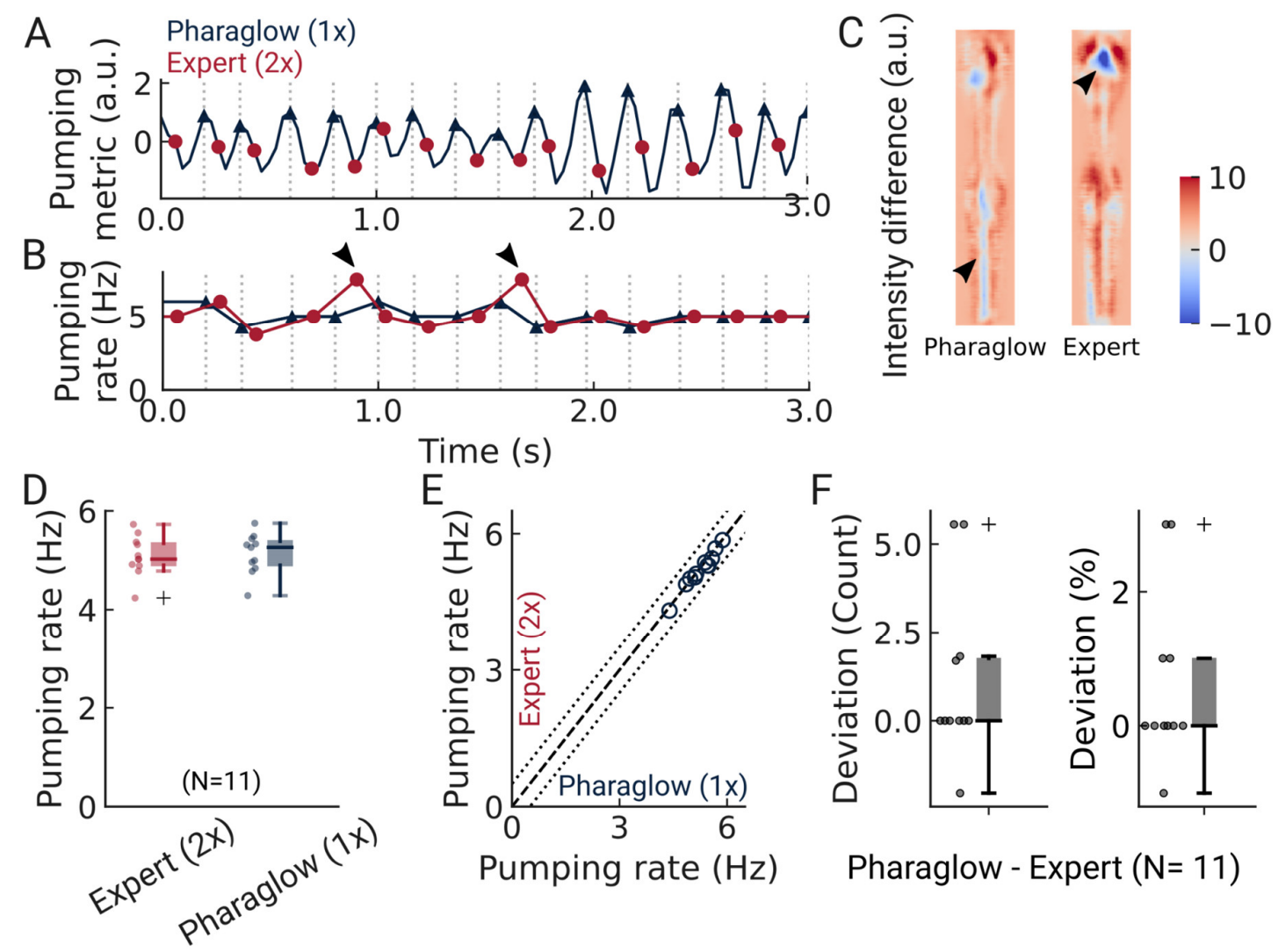

Figure 2. PharaGlow accurately detects pumping events in moving animals

(A) Example trace of the pumping metric (DV brightness variation) for a single animal on food (blue). Expert-annotated pumping events (red dots) and automatically detected events (blue triangles). (B) Pumping rate as determined by the expert (red) and PharaGlow (blue) corresponding to (A). (C) Difference between the images during detected pumps and non-pumps from (A) for the expert and pharaglow. Arrows highlight the lumen opening (Pharaglow) and terminal bulb contraction (expert). (D) Average pumping rates for 10 seconds each of expert counting (2x) and PharaGlow (1x) magnification for the same animals ( $N=11,10$ seconds of recording each). ( $E$ ) Correlation between the average pumping rates for the expert and Pharaglown $(N=11)$. ( $F)$ Deviation of the number of events between the expert and PharaGlow in \% or as absolute number of events in 10 seconds. 


\section{Developmental pumping}

During development, the pharynx grows with the body ${ }^{43}$, but the ratio between pharynx and body length decreases from L1 stage to adulthood ${ }^{15}$. To investigate how pumping rates change during development, we imaged cohorts of synchronized worms consecutively over three days in the middle of each of the four larval stages and as young adults. We accounted for the growing pharynx by adapting the magnification of our imaging system to achieve approximately the same spatial sampling of the pharynx at each stage (Fig. 3A-C). Animals were imaged directly on their culturing plates, and moved freely in the field of view (Fig. 3A), which allowed us to sample more than 150 trajectories per developmental stage with a total of more than 1000 animal tracks, which remained after filtering (see Methods). We filter animals that spend less than 1 minute in the field of view, leading to over-proportionally reducing young adult trajectories since these animals traverse the field of view very quickly despite the spatially proportional scaling. Nevertheless, we obtain large samples of animals due to new animals continually entering.

We find that on-food pumping rates increase slightly over the course of the larval stages, but much less dramatically than the velocity (Fig. 3D-F). Owing to time resolution and the large number of individual worms that can be analyzed using PharaGlow, it is possible to generate smooth probability density functions of pumping across the different larval stages (Fig. 3G). Some animals did not show pumping during our recording. It is possible that these animals show satiety quiescence ${ }^{24,44,45}$. Alternatively, we might have captured animals during lethargus, the period of sleep preceding each molt. However, this is unlikely because populations were synchronized and the imaging time points were chosen in the middle of each larval stage. Additionally, lethargus is incompatible with the observed distribution of velocities. We also find that similar to off-food reversals, which are constant throughout larval development ${ }^{46}$, on-food reversals occur at the same rates in all larval stages. However, while reversal rates between adults and larvae differ in the absence of food ${ }^{46}$, we find no significant difference in the reversal rate between young adults and larvae on-food (Fig. S3.1).

C. elegans are known to sense and react to light by initiating reversals and suppressing pumping. These reactions occur more frequently at short wavelengths and high power densities ${ }^{47,48,49}$. To determine if our imaging conditions affected behavior, we measured the light intensity and the leaving rates of animals during imaging. We used excitation light centered at $500 \mathrm{~nm}$, and measured an effective intensity of only $0.24 \mathrm{~mW} / \mathrm{mm}^{2}$ in the field of 
view, 54 times lower than the reported intensity that induces pumping inhibition or spitting ${ }^{49}$. We observed $5-25 \%$ of animals leaving the field of view during recordings, indicating a mild avoidance reaction which depended on the developmental stage (Fig. S3.2). Over longer time scales, exposure to light can reduce the worms' lifespan ${ }^{50}$. To control for photo-toxic effects, we split our developmental cohort into two groups. One group of animals was imaged consecutively at each larval stage (multiple exposures), the other group was left to grow under the same conditions, but only ever imaged once (single exposure).

We find that during all larval stages, the behavioral results of the two groups are similar, but not in young adults (Fig. S3.3). For the young adult cohort, the animals that were repeatedly imaged show a higher velocity compared to the single-exposure group, as well as differences in all other behavioral metrics we report. We speculate that this could be due to differences in drying of the plates during repeated imaging, different remaining food levels or a possible light-induced effect. To further assess the impact of the excitation light on behavior, we measured on-food pumping in adult animals expressing the red fluorophore mCherry compared to the strain expressing YFP, which is used throughout the paper. If the impact of the wavelength is non-negligible, the red fluorophore mCherry (Excitation centered at $587 \mathrm{~nm}$ ) should result in fewer reversals or accelerations compared to YFP, as these responses are wave-length dependent $47,48,49$. While we observe differences in the velocity and overall behavior with the blue light exposed animals, namely moving faster and exhibiting less dwelling (Fig. S3.4A), we find that pumping rates between the two populations are not significantly different (Fig. S3.4B).

Overall, we find that our imaging approach can be adapted to larvae by increasing the magnification, and our analysis pipeline is capable of handling hundreds of animals. While there are deviations between the automated detection and human counted data (Fig. S3.5), we accurately detect both mean and individual rates for all stages, with a median of error between experts and our method of less than 10\%. Over the course of 3 days and 5 imaging sessions, more than 1000 animals were tracked, significantly more than can be achieved with comparable methods (Table 1). 


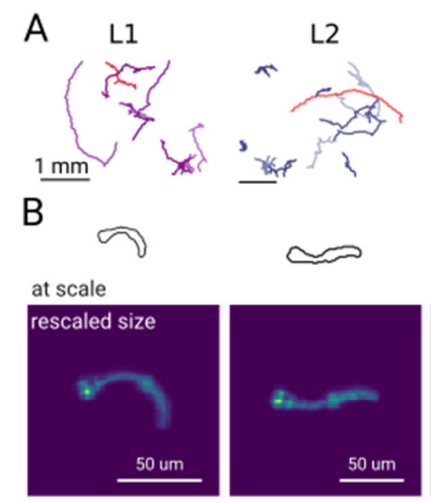

C
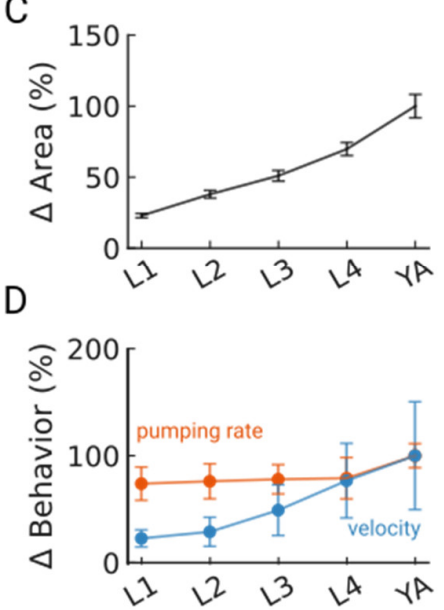

L3
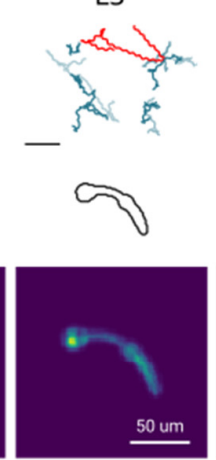

$\mathrm{E}$

要
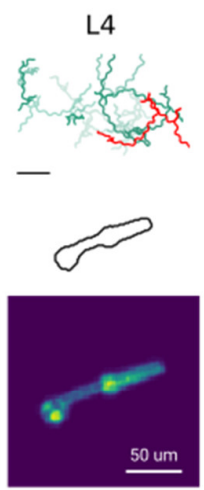

300
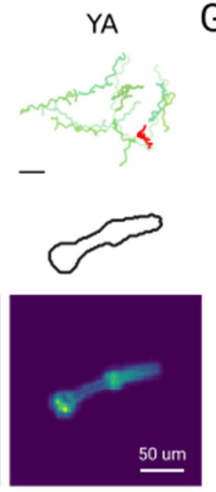

$50 \mathrm{um}$

G
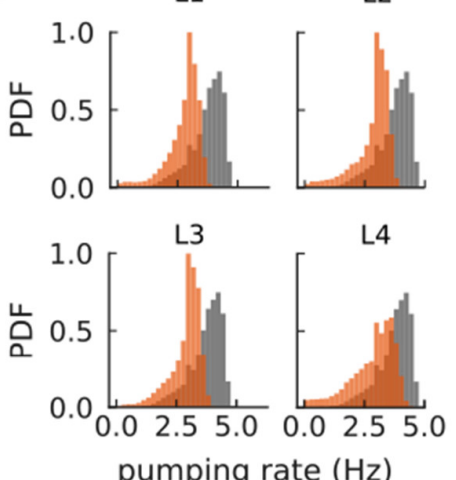

pumping rate $(\mathrm{Hz})$
F

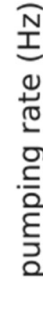

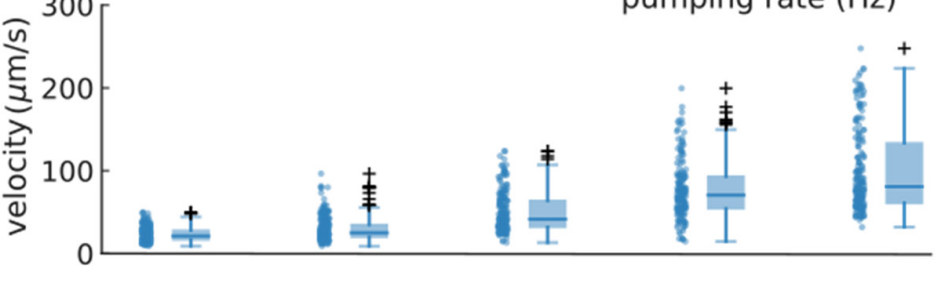

Figure 3. Changes in pumping and locomotion behavior during larval development

(A) Trajectories of 10 randomly selected animals at different larval stages (L1-L4) and young adults (YA). All scale bars correspond to $1 \mathrm{~mm}$ (top). (B) Size of the larvae and YA at the same scale (outlines, top) compared to the equal sizing achieved by adjusting the magnification (bottom). The image corresponds to the red track from (A)). (C) Relative change in the animal's area compared to the mean area of the YA stage. (D) Relative change in velocity (blue) and pumping rate (orange) across development compared to the mean of the YA stage. Error bars denote s.d. (E) time-averaged mean velocity and $(F)$ mean pumping rate for all animals. The boxplots follow Tukey's rule where the middle line indicates the median, the box denotes the first and third quartiles, and the whiskers show the 1.5 interquartile range above and below the box. (G) Pumping rate distribution for all larval stages as calculated by counting pumping events in a sliding window of width $=10 \mathrm{~s}$ and combining data from all animals of the same stage. The YA pumping rate distribution is underlaid in gray. The number of animals per condition is shown in (F). 


\section{Food intake is modulated by starvation}

Prior work showed that pumping rates off food are lower, but increase over the course of starvation and that this increase is mediated by a cholinergic pathway ${ }^{23}$. We confirm that pumping is dependent on the starvation duration, with a reduction in the pumping rate over the course of 3 hours (Fig. 4A-D). Beyond the first time point, our data are consistent with prior data ${ }^{23}$, showing a sustained rate of around $2-2.5 \mathrm{~Hz}$ (Fig. 4E). Previously, rates measured immediately after transferring worms off food (<30 min of starvation) were very low, possibly due to a lasting pumping suppression after harsh touch ${ }^{51}$, which we avoid by washing worms off plates instead of picking (see Methods).

Interestingly, for starved worms the distributions of both pumping rates and velocities show distinct sub-populations (Fig. 4F). For the earlier time points, a fraction of the animals show low speeds and pumping rates. At later times, the population becomes homogeneous with a well-defined mean rate and speed. When taken off food, $C$. elegans displays a restricted area search (local area search) which is characterized by frequent turns and reversals ${ }^{7,52-54}$. This behavior lasts between 30 - 60 min, after which animals switch to longer runs that cover more area. For the shortest starvation time point we sampled, we see a mixed population with distinct speeds and feeding rates, possibly reflecting some animals that are still performing a local search and others that are not. This is consistent with the fact that these distinct populations are not apparent in worm populations that stay on food (Fig. S4.1). 



Figure 4 Pumping is modulated by starvation

(A) Example trajectories of worms after 30 min starvation (blue) or 30 min continuously on food (red), $\mathrm{N}=10$. (B) The pumping rate distributions for the conditions in $(A)$ for all animals $\left(\mathrm{N}_{\text {starved }}=33\right.$, $N_{\text {onfood }}=111$ ). (C) Same as (A) but for animals starved, or kept on food for 210 min. (D) The pumping rate distributions corresponding to $\left(C ; N_{\text {starved }}=33, N_{\text {onfood }}=85\right)$. (E) Velocity, reversal rate, and pumping rate for animals starved and on-food controls. The sample size is given in the bottom panel. (F) Correlation between velocity and pumping rate for increasing starvation times. The cross indicates the mean (red) and standard deviation (white). The density is normalized by sample number. 


\section{Feeding mutants}

A desired capability for a high-throughput feeding tool is the ability to faithfully detect feeding rates in mutant animals which might have different pharyngeal contraction patterns and body motion, potentially increasing the difficulty of detecting feeding events. We therefore selected mutants with reported constitutively high (unc-31) or reduced (eat-18) feeding rates and different locomotory patterns. UNC-31 is involved in dense-core vesicle release, and unc-31 mutant animals display reduced, uncoordinated locomotion on food (Fig. 5A). We confirm that unc-31(e928) and unc-31(n1304) animals pump at rates comparable to wildtype, however, we see a bimodal distribution of rates with a fraction of animals showing markedly lower rates (Fig. 5B).

In contrast to unc-31, eat-18 mutant animals have no reported locomotor defects, but pump slower than wildtype ${ }^{28}$. EAT-18 is expressed in the pharyngeal muscle and interacts with a nAChR subunit EAT-2 to form a functional acetylcholine receptor ${ }^{27,55}$. Feeding impaired mutants were previously reported to have reduced body lengths and widths ${ }^{56}$. Expectedly, we found that eat-18 animals were smaller (Fig. 5C) and developed more slowly (approximately 91 hours from egg to adulthood compared to 63 hours for wildtype). While we detected pumping events at an average rate of $2 \mathrm{~Hz}$, the animals showed a different contraction pattern and timing than either unc-31 or wt animals (Fig. 5D, E). We confirmed that eat-18(ad820) animals lack the ability to perform fast pumping bursts (Fig.5B) and the duration of a pharyngeal contraction is about double compared to wt (Fig. 5E, S5.1 Video S2). We do observe a higher pumping rate than previously reported for eat-18, where animals were reported to rarely pump during experiments $\left(<0.5 \mathrm{~Hz},{ }^{27,28}\right)$. It is possible that some of the detected pumps in our measurements are either peristaltic movements or other nonproductive muscular motion. However, when verifying sample animals, we did observe slow pumping bursts at the $1-2 \mathrm{~Hz}$ rates indicated by PharaGlow, suggesting that these animals are able to pump at this frequency (Fig. 5E, arrows and Supplementary Video S2). We also found that eat-18 animals showed significantly fewer reversals than wildtype, indicating a role for the nAch receptor in modulating reversals. This is likely mediated by extrapharyngeally located neurons, since eat-18 is reported to show expression not only in the pharyngeal muscle, but also some unidentified somatic neurons ${ }^{28}$. 

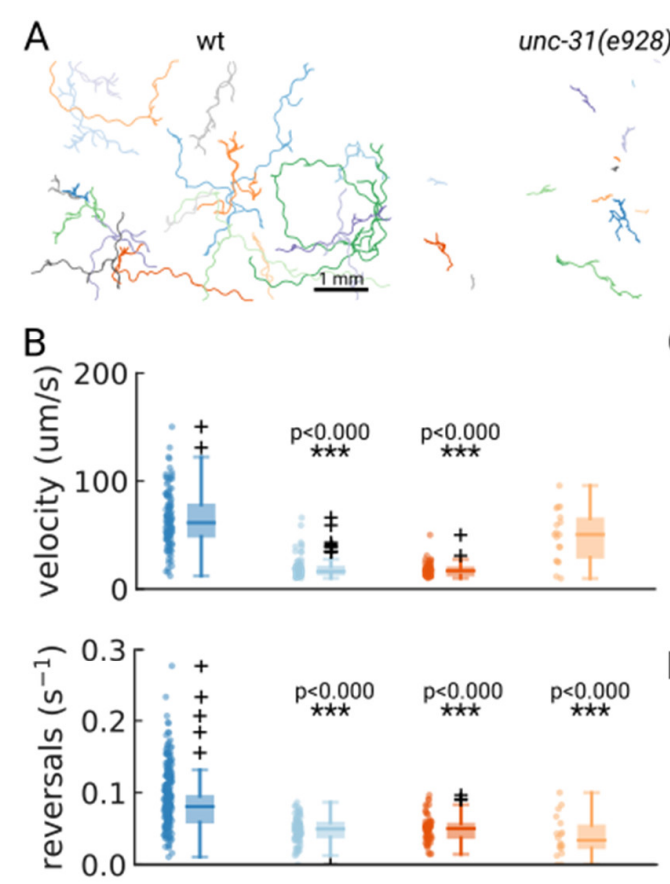

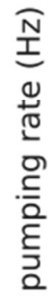

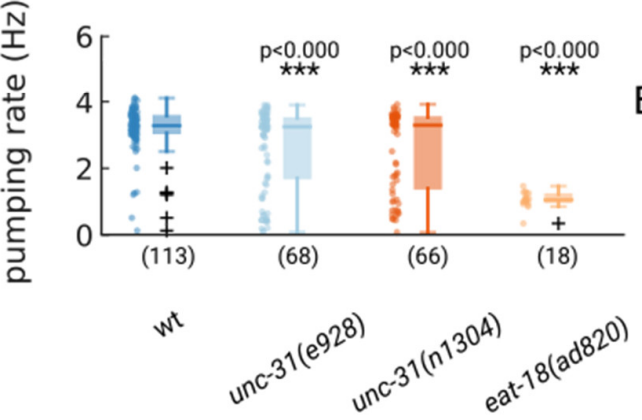

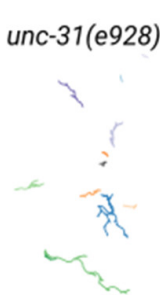

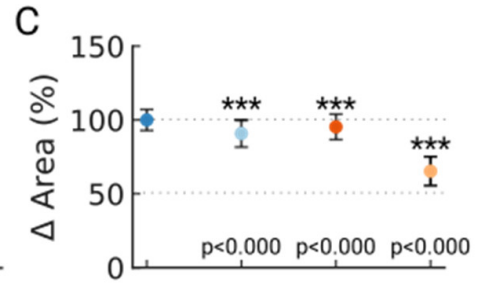

D $\Delta \mathrm{F}$
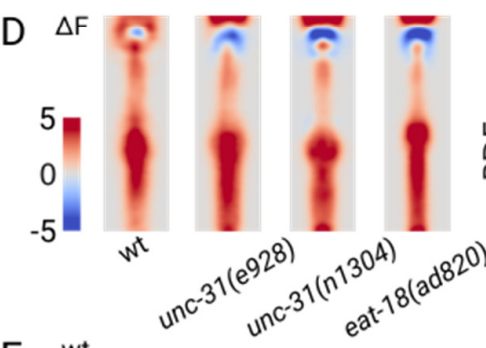

E wt

unc-31(e928)

unc-31(n1304)

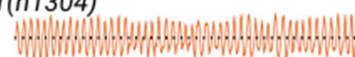

eat-18(ad820)

(.).

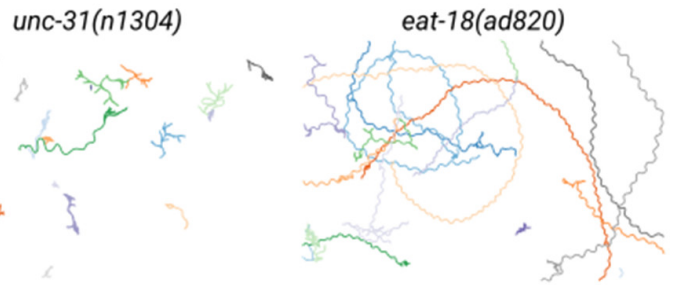

$\mathrm{F}$
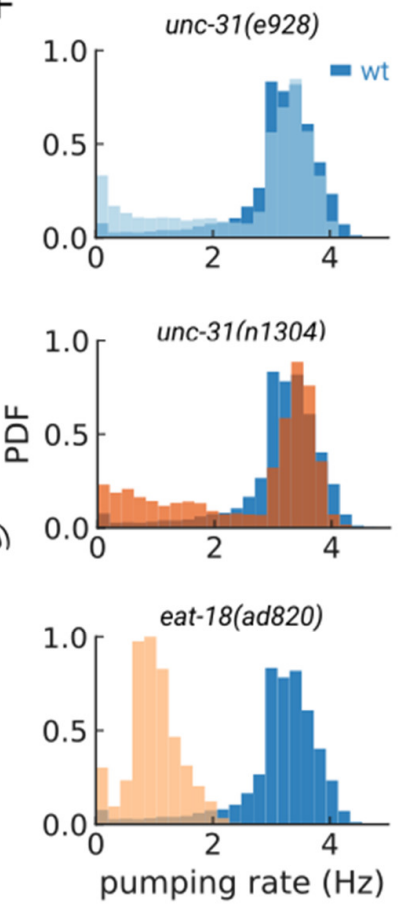

Figure 5 Automated pumping detection in feeding mutants

(A) Example trajectories of tracked animals $(\mathrm{N}=20$, except $\mathrm{N}=18$ for eat-18(ad820)). (B) Velocity, reversal rate and pumping rate for all genotypes. Significant differences between a mutant and wt are indicated as $* *(p<0.01)$ and $* \star \star(p<0.001)$, and exact $p$-values are reported in each panel for all $p$ values $<0.01$ (Welch's unequal variance two-tailed t-test). The sample size is given in parentheses in the bottom panel. (C) Mean and standard deviation of the pharyngeal areas relative to wt. (D) Mean change in myo-2p::YFP fluorescence during pumping averaged across a set of randomly chosen animals $(\mathrm{N}=10)$ and 100 randomly sampled pumping events per genotype. (E) Pumping metric for a representative sample animal per genotype. Arrows in the eat-18(ad820) trace denote slow contractions. (F) Pumping rate distributions. The wt pumping rate distribution is underlaid in dark blue. 


\section{Limitations and requirements}

Using a combination of low-magnification fluorescence imaging and dedicated analysis software, we show that it is possible to perform high-throughput, automated pumping detection of worms crawling on standard culture plates. There are some limitations to the method that are due to the reliance on a fluorescent indicator in the pharyngeal muscle and the handling of the large datasets that are generated. We find that uneven plates or improper focus leads to low signal-to-noise ratios. With careful focusing, imaging the center of evenly poured plates and using our custom peak detection method that is adaptive to the image quality (see Methods), these pitfalls can be mediated. Additionally, once at focus, small variations of the worm height do not affect the result, as low-magnification imaging results in a large depth of field.

As it is necessary for our approach to label the pharynx, mutant characterization with our tool would require crossing all possible mutants with a fluorescent reporter strain, which might be labor intensive but is nonetheless still a standard genetic practice when a reporter is used. While most experiments in this paper were performed with a homozygous, integrated reporter background (gnals1), we have also used extrachromosomal arrays with success (Fig. S3.4), which allows the use of animals that have a myo-2 reporter as a coinjection marker, for example. In addition, since our tool relies on the detection of fluorescent protein, siblings losing the transgene on the plate will not interfere with the analysis.

To maximize the field of view, we have chosen the smallest spatial resolution at which we could reliably detect pumping in wildtype adults. To ensure detection in smaller animals, increasing the magnification in these cases is recommended. The final requirement is related to data management and handling. While the hardware requirements are relatively straightforward and only a fluorescence dissecting microscope and a Megapixel camera are needed, the data rate of the large area scan cameras is $>6 \mathrm{~GB} / \mathrm{s}$. While the analysis can be run on a laptop or desktop computer, runtime is much improved when using a computing cluster.

\section{Discussion}

We developed a microscopy protocol and customized image analysis software that enables simultaneous measurements of locomotion and feeding in unrestrained animals. We are able to accurately detect feeding rates in populations of animals on plates at single-worm, 
single-pump resolution. Our fully automatic method provides an increase in throughput by more than one order of magnitude, and does not require any laborious handling of the animals or microfluidic devices. As our imaging does not adversely affect worms, animals can be imaged multiple times, as we demonstrated by following pumping in a population of developing animals. By enabling measurements of unrestrained animals directly on agarose plates, our approach creates opportunities for studying novel behaviors, such as foraging in complex environments ${ }^{9,35}$ or behavioral coordination ${ }^{31,57}$.

To understand the neural basis of feeding, resolving the decisions underlying feeding behavior is required ${ }^{12,13}$. However, bulk methods capable of extracting population averages of feeding rates are insufficient to understand underlying neural activity, as the data lack the temporal resolution to correlate feeding behavior with neural activity. Additionally, it is desirable to image animals in their normal culturing environments to compare feeding behaviors to established baselines e.g., for locomotion and reversals. Our method enables acquisition of feeding behavior in $C$. elegans for multiple animals at the same time.

An essential requirement for broad applicability of such a tool is its use for genetic and pharmacological screens. We demonstrated the suitability of our tool for studying the pumping and locomotory behaviors of unc-31 and eat-18 animals. We could identify a bimodal distribution of pumping rates for unc-31 mutants, which indicates that while these animals are capable of fast pumping, they do not show the same temporal regulation as N2 animals which show rapid transitions between slow and fast pumping, and pump fast most of the time at high food levels ${ }^{13}$. We could also reveal a previously unreported alteration in pump duration for eat-18 animals. Surprisingly, during confirmation of the feeding defect, we also discovered a previously unreported locomotion defect, hinting at a broader function for eat-18 possibly outside of the pharynx. Additionally, PharaGlow enables easy pharmacological screens on plates, by allowing experimenters to directly apply compounds to culture plates without requiring microfluidics or immobilization. Immobilization often requires the addition of serotonin (5-HT) to stimulate pumping, as pumping is suppressed in restrained animals ${ }^{32}$. It is likely that observed feeding defects differ between crawling, foodstimulated animals and serotonin-stimulated animals ${ }^{20}$, and that new experiments will be effective in identifying phenotypes that went unnoticed in immobilized preparations.

To mimic realistic foraging situations would require extending the foraging arena by supplying variable patches of food, and providing a more interesting landscape rather than a 
homogenous 2D plate environment. Patch foraging has been extensively studied in $C$. elegans, both with respect to entry into single patches $8,9,12$ and food choice ${ }^{12,58,59}$, though these studies focused on locomotion rather than feeding rates. Testing models of patch foraging and the predictions made from such models ${ }^{60}$ while including measured food intake explicitly will help better define the limits in which simple models of foraging are applicable, and will ideally allow extension to the underlying strategies learned or inherited by worms living in different environments.

Recent work has succeeded in building a scaffolded 3D environment where $C$. elegans dauer larvae were observed ${ }^{61}$, indicating that behaviors are richer than in the typical 2D culturing environments. Our method, combined with animal tracking in 3D, could enable assays for feeding in these naturalistic contexts, while traditional grinder-tracking optical methods would likely be difficult due to the size and orientation changes of the pharynx in 3D. Technically, current limitations of the imaging, such as the field of view can be extended by using larger camera arrays, which will enable scanning across multiple centimeter-sized fields of view.

A further extension of this work would be to image feeding activity in related species, either Caenorhabditis nematodes collected from different field sites, or even predatory nematodes like Pristionchus pacificus, which has a bacterial feeding mode similar to $C$. elegans and additionally a predatory mode when killing the larvae of other nematodes. While the requirement for labeling the muscle is a prerequisite of our method, the myo- 2 gene is a myosin heavy chain that is conserved among nematodes and will likely show similar expression in closely related species. Alternatively, labeling parts of the pharynx with lipophilic dyes would be a possibility to extend the usage of this tool beyond species that are genetically tractable. Overall, our tool provides the ability to study feeding behaviors that will create new views into worm behavior. 


\section{Methods}

\begin{tabular}{|c|c|c|c|c|}
\hline \multicolumn{5}{|l|}{ Key Resources Table } \\
\hline $\begin{array}{l}\text { Reagent type } \\
\text { (species) or } \\
\text { resource }\end{array}$ & Designation & $\begin{array}{l}\text { Source or } \\
\text { reference }\end{array}$ & Identifiers & $\begin{array}{l}\text { Additional } \\
\text { information }\end{array}$ \\
\hline $\begin{array}{l}\text { strain, strain } \\
\text { background } \\
\text { (Escherichia coli } \\
\text { OP50) }\end{array}$ & OP50 & CGC & CGC:OP50 & \\
\hline $\begin{array}{l}\text { Recombinant DNA } \\
\text { reagent }\end{array}$ & $\begin{array}{l}\text { myo- } \\
\text { 2p::mCherry::unc-54 } \\
\text { 3'utr }\end{array}$ & Addgene & pCFJ90 & $\begin{array}{l}5 \mathrm{ng} / \mathrm{ul} \\
\text { injected into } \\
\text { N2 }\end{array}$ \\
\hline $\begin{array}{l}\text { Recombinant DNA } \\
\text { reagent }\end{array}$ & pPHA2::GFP-F & $\begin{array}{l}\text { Gift from } \\
\text { Marc Pilon }\end{array}$ & pMS17 & $\begin{array}{l}50 \mathrm{ng} / \mathrm{ul} \\
\text { injected into } \\
\mathrm{N} 2\end{array}$ \\
\hline $\begin{array}{l}\text { strain, strain } \\
\text { background } \\
\text { (C. elegans) }\end{array}$ & N2 & CGC & N2 & $\begin{array}{l}\text { Background } \\
\text { for INF30 }\end{array}$ \\
\hline $\begin{array}{l}\text { strain, strain } \\
\text { background } \\
\text { (C. elegans) }\end{array}$ & gnals1 [myo-2p:::yfp] & CGC & GRU101 & \\
\hline $\begin{array}{l}\text { strain, strain } \\
\text { background } \\
\text { (C. elegans) }\end{array}$ & $\begin{array}{c}\text { nonEx9[pPHA2::GFP- } \\
\text { F myo- } \\
\text { 2p::mCherry::unc-54 } \\
\text { 3'utr] }\end{array}$ & $\begin{array}{l}\text { This } \\
\text { publication }\end{array}$ & INF30 & $\begin{array}{l}5 \mathrm{ng} / \mathrm{ul} \text { of } \\
\text { pMS17 and } 5 \\
\text { ng/ul of } \\
\text { pCFJ90 } \\
\text { injected into } \\
\text { N2 }\end{array}$ \\
\hline $\begin{array}{l}\text { strain, strain } \\
\text { background } \\
\text { (C. elegans) }\end{array}$ & $\begin{array}{c}\text { unc-31(e928) gnals1 } \\
\text { IV }\end{array}$ & $\begin{array}{l}\text { This } \\
\text { publication }\end{array}$ & INF5 & $\begin{array}{l}\text { Cross of unc- } \\
31(e 928) \text { with } \\
\text { GRU101 }\end{array}$ \\
\hline $\begin{array}{l}\text { strain, strain } \\
\text { background } \\
\text { (C. elegans) }\end{array}$ & $\begin{array}{c}\text { unc-31(n1304) } \\
\text { gnals1 IV }\end{array}$ & $\begin{array}{l}\text { This } \\
\text { publication }\end{array}$ & INF17 & $\begin{array}{l}\text { Cross of unc- } \\
31(n 1304) \\
\text { with GRU101 }\end{array}$ \\
\hline $\begin{array}{l}\text { strain, strain } \\
\text { background } \\
\text { (C. elegans) }\end{array}$ & $\begin{array}{l}\text { eat-18(ad820) l; } \\
\text { gnals1 IV }\end{array}$ & $\begin{array}{l}\text { This } \\
\text { publication }\end{array}$ & INF44 & $\begin{array}{l}\text { Cross of eat- } \\
\text { 18(ad820) } \\
\text { with GRU101 }\end{array}$ \\
\hline $\begin{array}{l}\text { software, } \\
\text { algorithm }\end{array}$ & PharaGlow & $\begin{array}{l}\text { This } \\
\text { publication }\end{array}$ & & \\
\hline
\end{tabular}




\section{C. elegans maintenance}

C. elegans were grown on NGM plates at $20^{\circ} \mathrm{C}$. Worms were synchronized by letting adult gravid animals lay eggs for $2-3 \mathrm{~h}$, then removing the adults. The average time from egg to young adult stage for strain GRU101 (gnals1[myo-2p::yfp]) was 63h. Before the experiment, synchronized adults were washed off the culture plates with $1 \mathrm{ml}$ of $\mathrm{M} 9$ and collected in an Eppendorf tube. Worms were allowed to settle for $1 \mathrm{~min}$, the supernatant was removed and the tube was refilled with $\mathrm{M9}$. Washing was repeated two more times. The washing was sufficient in that we did not observe animals remaining in the spots containing the remainder of M9 on the assay plates, suggesting that the bacterial amount was too diluted to induce dwelling behavior.

\section{Strains}

\begin{tabular}{|c|c|c|c|}
\hline Strain & Genotype & $\begin{array}{l}\text { Figures } \\
\text { (Panels) }\end{array}$ & Source \\
\hline GRU101 & gnals1[myo-2p::yfp] & All & CGC \\
\hline INF30 & $\begin{array}{c}\text { nonEx9[pPHA2::GFP-F myo- } \\
2 p:: m C h e r r y:: u n c-54 \text { 3'utr] }\end{array}$ & Fig.S3.4 & This publication \\
\hline INF5 & unc-31(e928) gnals1 IV & Fig. 4 & This publication \\
\hline INF17 & unc-31(n1304) gnals1 IV & Fig. 4 & This publication \\
\hline INF44 & eat-18(ad820) l; gnals1 IV & Fig. 4 & This publication \\
\hline
\end{tabular}

\section{Imaging setup}

Imaging of worms at $1 \mathrm{x}$ magnification was performed using a commercial upright epifluorescence microscope (Axio Zoom V16; Zeiss) equipped with a 1x objective (PlanNeoFluar Z 1.0x/N.A. 0.25). For imaging of YFP fluorescence, light from an LED lamp (X-Cite XYLIS) was reflected towards the sample using a dichroic mirror (FT 515; Zeiss) and filtered (BP 500/25; Zeiss). Emitted light was filtered using a band-pass filter (BP 535/30; Zeiss) and focused onto the camera sensor (BASLER; acA3088-57um) using a camera adapter with an additional $0.5 x$ magnification $\left(60 \mathrm{~N}-\mathrm{C}_{2}^{2} / 3\right.$ " $0.5 \mathrm{x}$; Zeiss). The power density of fluorescence excitation at the focal plane $\left(0.24 \mathrm{~mW} / \mathrm{mm}^{2}\right.$ at $\left.500 \mathrm{~nm}\right)$ was measured using a power meter sensor (PS19Q; Coherent) with the corresponding controller (PowerMax; Coherent). Animals were imaged at $30 \mathrm{fps}$ for 5 minutes unless otherwise indicated. For imaging of mCherry, the filter cube was replaced with a commercial filter set (64 HE; Zeiss). The resulting power density using this cube was $0.76 \mathrm{~mW} / \mathrm{mm}^{2}$ at the focal plane. 


\section{Developmental pumping experiments}

Worms were pre-synchronized by hypochlorite bleaching, allowed to hatch overnight in M9 and then cultured on NGM plates with OP50 at $20^{\circ} \mathrm{C}$. On day 3 after pre-synchronization, worms were synchronized again by letting 20 gravid animals lay eggs for $2 \mathrm{~h}$ per assay plate, then removing the adults and letting embryos grow for specific durations to reach the appropriate larval or adult stage (19 $\mathrm{h}$ for mid-L1, $31 \mathrm{~h}$ for mid-L2, $39 \mathrm{~h}$ for mid-L3, $50 \mathrm{~h}$ for mid-L4, $65 \mathrm{~h}$ for young adults). For the assay plates, $40 \mu \mathrm{l}$ of $E$. coli OP50 culture was spotted onto an empty $6 \mathrm{~cm}$ NGM plate a few hours before the synchronization and left to dry. Synchronized worms were imaged directly on their assay plates as described in section "Imaging setup". The magnification for each stage was chosen to achieve an approximate pharynx length of $\sim 60$ pixels $(2 x(1.18 \mu \mathrm{m} / \mathrm{px})$ for $L 1,1.5 x(1.57 \mu \mathrm{m} / \mathrm{px})$ for $L 2,1.4 x(1.69$ $\mu \mathrm{m} / \mathrm{px})$ for $L 3,1.3 x(1.81 \mu \mathrm{m} / \mathrm{px})$ for $L 4$ and the standard $1 x(2.36 \mu \mathrm{m} / \mathrm{px})$ for young adults). 3 assay plates were imaged once per stage, and 3 additional plates were imaged at each stage to test for photo-sensitivity.

\section{Starvation experiments}

Washed animals were transferred to the center of an empty $6 \mathrm{~cm}$ NGM plate at room temperature and left to recover for 15 minutes before imaging. The same plate was imaged at defined time points for progressively more starved animals (at $30 \mathrm{~min}, 90 \mathrm{~min}, 150 \mathrm{~min}$ and 210 min after being taken off food). The field of view was chosen randomly on the plate but was required to contain at minimum 3 worms at the beginning of the recording. For control, washed animals were transferred close to a $40 \mu$ of $E$. coli OP50 lawn, which was spotted onto an empty $6 \mathrm{~cm}$ NGM plate a few hours before the recordings and allowed to dry. Acclimation time and recording are similar for starved animals.

\section{Automated Analysis}

Pharyngeal pumping - fluorescence data

Animals were tracked using our custom python analysis package PharaGlow which is freely available under a permissive GPL 3.0 license. In brief, pharaglow runs a three-step analysis: 1. center of mass tracking and collision detection, 2. linking detected objects to trajectories and 3. extracting centerline, contour, width, and other parameters of the shape to allow extracting pharyngeal pumping events. Tracking uses the soft matter package ${ }^{62}$. The code is fully modular and any existing tracking code could in principle be used for the first two steps provided the input data is formatted to pharaglow standards. We provide example 
data and example jupyter notebooks to help users make use of our package both in personal computer and high-performance cluster settings. The resulting files contain the position, and the straightened images which are further processed to extract the behavioral measures as described in Fig. 1 and section 'Pharyngeal pumping - postprocessing". '

\section{Pharyngeal pumping - postprocessing}

To obtain pumping traces from straightened animals, the inverted maximum of the dorsoventral standard deviation of brightness is calculated for each straightened frame per animal (Fig. 1E). This metric is sensitive to the opening of the pharyngeal lumen and terminal bulb contractions. Peaks in the resulting trace correspond to pumping events. Due to the animal motion, uneven illumination or defocusing can modify the baseline of the pumping metric. We correct for baseline fluctuations and spurious fluorescence changes by subtracting the background fluctuations using a rolling mean filter of $1 \mathrm{~s}$ and a smoothing filter of width $=$ $66 \mathrm{~ms}$ (2 frames). We detect peaks using AMPD, an algorithm for peak detection in quasiperiodic signals ${ }^{63}$. We also require the peak distances to obey physiologically reasonable rates i.e., the peaks can not be closer than $\mathrm{dmin}=132 \mathrm{~ms}$ ( 4 frames). To automatically establish the noise level of the trace, we compare the incidence of intervals between detected peaks that violate the assumption $d \min >132 \mathrm{~ms}$ and select the minimal prominence required, such that the fraction of violating intervals is lower than a sensitivity parameter $s$. For all datasets, we set $s=0.999$.

\section{Other behavioral parameters}

Velocity was calculated from the tracked center of mass of the labeled pharynx. Reversals were calculated based on the angle between the pharynx and the animal's nose tip direction. To avoid spurious reversals, the nose tip trajectories are coarse-grained to $6 \mathrm{~Hz}$, and the angle between the nose tip and pharynx is smoothed with a window of width $=1 \mathrm{~s}$ (30 frames). Reversals shorter than $0.5 \mathrm{~s}$ are removed. The estimation of the pharyngeal area is based on an automated threshold of the pharynx.

\section{Animal selection}

All animals that were successfully tracked for at least 30 seconds (Fig. 1,2,3) were included. No other filtering or outlier removal was performed. Due to synchronization, all animals in the field of view were of similar size in the wildtype experiments. For eat-18 mutants, the size and developmental stage of the animals were more dispersed and only animals that had the appropriate size for their stage were included (Fig. 5). In the starvation experiments, animals 
that were successfully tracked for at least 20 seconds were included due to the larger velocity in this condition (Fig. 4).

\section{Manual annotation of pumping behavior}

Movies for individual animals were created from a large field of view and expert annotators counted pumps by displaying the movie using the cell counter tool in Fiji ${ }^{64}$. The annotators were blinded to the movie conditions and to the other experts' results. For comparing annotators at $2 x$ magnifications, movies were recorded at the higher magnification and downscaled to $1 x$ by binning.

Table 1: Comparison of classes of automated methods for pumping analysis

\begin{tabular}{|l|l|l|l|l|l|l|l|}
\hline Technique & $\begin{array}{l}\text { Single } \\
\text { pump }\end{array}$ & $\begin{array}{l}\text { Single } \\
\text { worm }\end{array}$ & $\begin{array}{l}\text { Animals/ } \\
\text { setup }\end{array}$ & Method & Label & Constrained? & Source \\
\hline $\begin{array}{l}\text { Bioluminescent } \\
\text { bacteria }\end{array}$ & No & No & $100-1000$ & Microscopy & No & No & 35 \\
\hline $\begin{array}{l}\text { Luciferase } \\
\text { expressing } \\
\text { worms }\end{array}$ & No & Yes & 100 & Microscopy & Yes & No & 65 \\
\hline Optical density & No & No & $100-1000$ & Absorption & No & No & 38 \\
\hline $\begin{array}{l}\text { Tracking } \\
\text { microscope }\end{array}$ & Yes & Yes & 1 & Microscopy & No & No & $30,31,39$ \\
\hline pWarp & Yes & Yes & 4 & Microscopy & No & microfluidic & 19 \\
\hline NemaChip & Yes & Yes & 8 & $\begin{array}{l}\text { Electrophysiology } \\
\text { / EPG }\end{array}$ & No & microfluidic & 40 \\
\hline PharaGlow & Yes & Yes & $\mathbf{3 0 - 5 0}$ & Microscopy & Yes & No & $\begin{array}{l}\text { This } \\
\text { paper }\end{array}$ \\
\hline
\end{tabular}

\section{Acknowledgments}

We thank James Lightfoot for helpful comments. Some strains were provided by the CGC, which is funded by NIH Office of Research Infrastructure Programs (P40 OD010440). Some figure panels were created with biorender.com. We thank Marc Pilon for giving us a plasmid.

\section{Data and Code availability statement}

The data from this manuscript is available at https://osf.io/fy4ed/. The Code repository for the PharaGlow package can be found at https://github.com/scholz-lab/PharaGlow. 


\section{References}

1. Buehlmann, C., Mangan, M. \& Graham, P. Multimodal interactions in insect navigation. Anim. Cogn. 23, 1129-1141 (2020).

2. Yoon, T., Geary, R. B., Ahmed, A. A. \& Shadmehr, R. Control of movement vigor and decision making during foraging. Proceedings of the National Academy of Sciences 115, E10476-E10485 (2018).

3. Nonacs, P. State dependent behavior and the Marginal Value Theorem. Behav. Ecol. 12, $71-83$ (2001).

4. Asahina, K., Pavlenkovich, V. \& Vosshall, L. B. The survival advantage of olfaction in a competitive environment. Curr. Biol. 18, 1153-1155 (2008).

5. Corrales-Carvajal, V. M., Faisal, A. A. \& Ribeiro, C. Internal states drive nutrient homeostasis by modulating exploration-exploitation trade-off. eLife 5, (2016).

6. Ghosh, D. D. et al. Neural Architecture of Hunger-Dependent Multisensory Decision Making in C. elegans. Neuron 92, 1049-1062 (2016).

7. Gray, J. M., Hill, J. J. \& Bargmann, C. I. A circuit for navigation in Caenorhabditis elegans. Proc. Natl. Acad. Sci. U. S. A. 102, 3184-3191 (2005).

8. Flavell, S. W. et al. Serotonin and the neuropeptide PDF initiate and extend opposing behavioral states in C. elegans. Cell 154, 1023-1035 (2013).

9. Iwanir, S. et al. Serotonin promotes exploitation in complex environments by accelerating decision-making. BMC Biol. 14, 9 (2016).

10. Zjacic, N. \& Scholz, M. The role of food odor in invertebrate foraging. Genes Brain Behav. e12793 (2022).

11. Avery, L. \& You, Y.-J. C. elegans feeding. WormBook 1-23 (2012).

12. Katzen, A. et al. The nematode worm $C$. elegans chooses between bacterial foods exactly as if maximizing economic utility. bioRxiv 2021.04.25.441352 (2021) doi:10.1101/2021.04.25.441352. 
13. Scholz, M., Dinner, A. R., Levine, E. \& Biron, D. Stochastic feeding dynamics arise from the need for information and energy. Proc. Natl. Acad. Sci. U. S. A. 114, 9261-9266 (2017).

14. Seymour, M. K., Wright, K. A. \& Doncaster, C. C. The action of the anterior feeding apparatus of Caenorhabditis elegans (Nematoda: Rhabditida). J. Zool. 201, 527-539 (1983).

15. Avery, L. \& Shtonda, B. B. Food transport in the C. elegans pharynx. J. Exp. Biol. 206, 2441-2457 (2003).

16. Fang-Yen, C., Avery, L. \& Samuel, A. D. T. Two size-selective mechanisms specifically trap bacteria-sized food particles in Caenorhabditis elegans. Proc. Natl. Acad. Sci. U. S. A. 106, 20093-20096 (2009).

17. Albertson, D. G. \& Thomson, J. N. The pharynx of Caenorhabditis elegans. Philos. Trans. R. Soc. Lond. B Biol. Sci. 275, 299-325 (1976).

18. Song, B.-M. \& Avery, L. Serotonin activates overall feeding by activating two separate neural pathways in Caenorhabditis elegans. J. Neurosci. 32, 1920-1931 (2012).

19. Scholz, M., Lynch, D. J., Lee, K. S., Levine, E. \& Biron, D. A scalable method for automatically measuring pharyngeal pumping in C. elegans. J. Neurosci. Methods 274, 172-178 (2016).

20. Lee, K. S. et al. Serotonin-dependent kinetics of feeding bursts underlie a graded response to food availability in C. elegans. Nat. Commun. 8, 14221 (2017).

21. Song, B.-M., Faumont, S., Lockery, S. \& Avery, L. Recognition of familiar food activates feeding via an endocrine serotonin signal in Caenorhabditis elegans. eLife 2, e00329 (2013).

22. Avery, L. \& Horvitz, H. R. Effects of starvation and neuroactive drugs on feeding in Caenorhabditis elegans. J. Exp. Zool. 253, 263-270 (1990).

23. You, Y.-J., Kim, J., Cobb, M. \& Avery, L. Starvation activates MAP kinase through the 
muscarinic acetylcholine pathway in Caenorhabditis elegans pharynx. Cell Metab. 3, $237-245$ (2006).

24. You, Y.-J., Kim, J., Raizen, D. M. \& Avery, L. Insulin, cGMP, and TGF-beta signals regulate food intake and quiescence in C. elegans: a model for satiety. Cell Metab. 7, 249-257 (2008).

25. Gruninger, T. R., Gualberto, D. G., LeBoeuf, B. \& Garcia, L. R. Integration of male mating and feeding behaviors in Caenorhabditis elegans. J. Neurosci. 26, 169-179 (2006).

26. Trojanowski, N. F., Raizen, D. M. \& Fang-Yen, C. Pharyngeal pumping in Caenorhabditis elegans depends on tonic and phasic signaling from the nervous system. Sci. Rep. 6, 22940 (2016).

27. Raizen, D. M., Lee, R. Y. \& Avery, L. Interacting genes required for pharyngeal excitation by motor neuron MC in Caenorhabditis elegans. Genetics 141, 1365-1382 (1995).

28. McKay, J. P., Raizen, D. M., Gottschalk, A., Schafer, W. R. \& Avery, L. eat-2 and eat-18 are required for nicotinic neurotransmission in the Caenorhabditis elegans pharynx. Genetics 166, 161-169 (2004).

29. Raizen, D. M. \& Avery, L. Electrical activity and behavior in the pharynx of Caenorhabditis elegans. Neuron 12, 483-495 (1994).

30. Li, Z. et al. Dissecting a central flip-flop circuit that integrates contradictory sensory cues in C. elegans feeding regulation. Nat. Commun. 3, 776 (2012).

31. Cermak, N. et al. Whole-organism behavioral profiling reveals a role for dopamine in state-dependent motor program coupling in C. elegans. eLife 9, (2020).

32. Takahashi, M. \& Takagi, S. Optical silencing of body wall muscles induces pumping inhibition in Caenorhabditis elegans. PLoS Genet. 13, e1007134 (2017).

33. Ramot, D., Johnson, B. E., Berry, T. L., Jr, Carnell, L. \& Goodman, M. B. The Parallel Worm Tracker: a platform for measuring average speed and drug-induced paralysis in nematodes. PLoS One 3, e2208 (2008). 
34. Swierczek, N. A., Giles, A. C., Rankin, C. H. \& Kerr, R. A. High-throughput behavioral analysis in C. elegans. Nat. Methods 8, 592-598 (2011).

35. Ding, S. S., Romenskyy, M., Sarkisyan, K. S. \& Brown, A. E. X. Measuring Caenorhabditis elegans Spatial Foraging and Food Intake Using Bioluminescent Bacteria. Genetics 214, 577-587 (2020).

36. Andersen, E. C., Bloom, J. S., Gerke, J. P. \& Kruglyak, L. A variant in the neuropeptide receptor npr-1 is a major determinant of Caenorhabditis elegans growth and physiology. PLoS Genet. 10, e1004156 (2014).

37. Kiyama, Y., Miyahara, K. \& Ohshima, Y. Active uptake of artificial particles in the nematode Caenorhabditis elegans. J. Exp. Biol. 215, 1178-1183 (2012).

38. Gomez-Amaro, R. L. et al. Measuring Food Intake and Nutrient Absorption in Caenorhabditis elegans. Genetics 200, 443-454 (2015).

39. Zou, W. et al. Decoding the intensity of sensory input by two glutamate receptors in one C. elegans interneuron. Nat. Commun. 9, 4311 (2018).

40. Lockery, S. R. et al. A microfluidic device for whole-animal drug screening using electrophysiological measures in the nematode C. elegans. Lab Chip 12, 2211-2220 (2012).

41. Okkema, P. G., Harrison, S. W., Plunger, V., Aryana, A. \& Fire, A. Sequence requirements for myosin gene expression and regulation in Caenorhabditis elegans. Genetics 135, 385-404 (1993).

42. Okkema, P. G. \& Fire, A. The Caenorhabditis elegans NK-2 class homeoprotein CEH-22 is involved in combinatorial activation of gene expression in pharyngeal muscle. Development 120, 2175-2186 (1994).

43. Knight, C. G., Patel, M. N., Azevedo, R. B. R. \& Leroi, A. M. A novel mode of ecdysozoan growth in Caenorhabditis elegans. Evol. Dev. 4, 16-27 (2002).

44. Gallagher, T., Kim, J., Oldenbroek, M., Kerr, R. \& You, Y.-J. ASI regulates satiety 
quiescence in C. elegans. J. Neurosci. 33, 9716-9724 (2013).

45. Davis, K. C., Choi, Y.-I., Kim, J. \& You, Y.-J. Satiety behavior is regulated by ASI/ASH reciprocal antagonism. Sci. Rep. 8, 6918 (2018).

46. Chiba, C. M. \& Rankin, C. H. A developmental analysis of spontaneous and reflexive reversals in the nematode Caenorhabditis elegans. J. Neurobiol. 21, 543-554 (1990).

47. Ward, A., Liu, J., Feng, Z. \& Xu, X. Z. S. Light-sensitive neurons and channels mediate phototaxis in C. elegans. Nat. Neurosci. 11, 916-922 (2008).

48. Bhatla, N., Droste, R., Sando, S. R., Huang, A. \& Horvitz, H. R. Distinct Neural Circuits Control Rhythm Inhibition and Spitting by the Myogenic Pharynx of C. elegans. Curr. Biol. 25, 2075-2089 (2015).

49. Bhatla, N. \& Horvitz, H. R. Light and hydrogen peroxide inhibit C. elegans Feeding through gustatory receptor orthologs and pharyngeal neurons. Neuron $85,804-818$ (2015).

50. De Magalhaes Filho, C. D. et al. Visible light reduces $C$. elegans longevity. Nat. Commun. 9, 927 (2018).

51. Keane, J. \& Avery, L. Mechanosensory inputs influence Caenorhabditis elegans pharyngeal activity via ivermectin sensitivity genes. Genetics $164,153-162$ (2003).

52. Hills, T., Brockie, P. J. \& Maricq, A. V. Dopamine and glutamate control area-restricted search behavior in Caenorhabditis elegans. J. Neurosci. 24, 1217-1225 (2004).

53. Sawin, E. R., Ranganathan, R. \& Horvitz, H. R. C. elegans locomotory rate is modulated by the environment through a dopaminergic pathway and by experience through a serotonergic pathway. Neuron 26, 619-631 (2000).

54. Calhoun, A. J., Chalasani, S. H. \& Sharpee, T. O. Maximally informative foraging by Caenorhabditis elegans. eLife 3, e04220 (2014).

55. Choudhary, S. et al. EAT-18 is an essential auxiliary protein interacting with the nonalpha nAChR subunit EAT-2 to form a functional receptor. PLoS Pathog. 16, e1008396 
(2020).

56. Mörck, C. \& Pilon, M. C. elegans feeding defective mutants have shorter body lengths and increased autophagy. BMC Dev. Biol. 6, 39 (2006).

57. Hardaker, L. A., Singer, E., Kerr, R., Zhou, G. \& Schafer, W. R. Serotonin modulates locomotory behavior and coordinates egg-laying and movement in Caenorhabditis elegans. J. Neurobiol. 49, 303-313 (2001).

58. Milward, K., Busch, K. E., Murphy, R. J., de Bono, M. \& Olofsson, B. Neuronal and molecular substrates for optimal foraging in Caenorhabditis elegans. Proc. Natl. Acad. Sci. U. S. A. 108, 20672-20677 (2011).

59. Dal Bello, M., Pérez-Escudero, A., Schroeder, F. C. \& Gore, J. Inversion of pheromone preference optimizes foraging in C. elegans. eLife 10, (2021).

60. Davidson, J. D. \& Hady, A. E. Foraging as an evidence accumulation process. PLoS Comput. Biol. 15, e1007060 (2019).

61. Guisnet, A., Maitra, M., Pradhan, S. \& Hendricks, M. A three-dimensional habitat for $C$. elegans environmental enrichment. PLoS One 16, e0245139 (2021).

62. Allan, D. et al. soft-matter/trackpy: Trackpy v0. 4.2. Zenodo, DOI 10, (2019).

63. Scholkmann, F., Boss, J. \& Wolf, M. An Efficient Algorithm for Automatic Peak Detection in Noisy Periodic and Quasi-Periodic Signals. Algorithms 5, 588-603 (2012).

64. Schindelin, J. et al. Fiji: an open-source platform for biological-image analysis. Nat. Methods 9, 676-682 (2012).

65. Rodríguez-Palero, M. J. et al. An automated method for the analysis of food intake behaviour in Caenorhabditis elegans. Sci. Rep. 8, 3633 (2018). 


\section{Supplementary Figures}

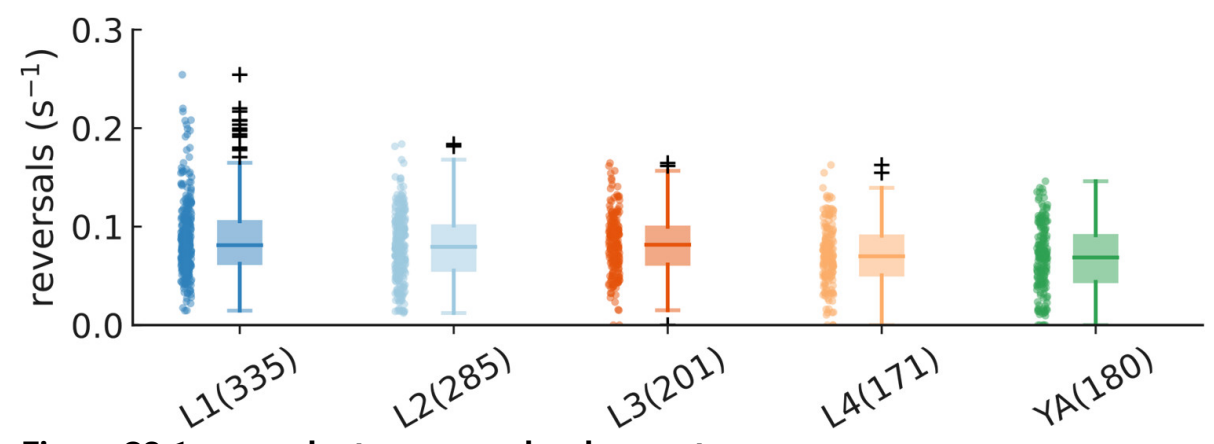

Figure S3.1 reversal rates across development.

Reversal rates throughout development are detected by comparing the nose-tip motion to the center of mass motion. Number of animals is shown in parenthesis.

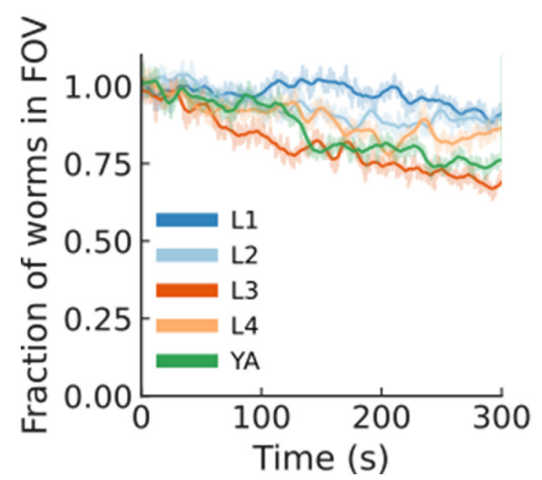

Figure S3.2 Only a mild leaving was induced by light

Mean number of animals in the field of view during the recording (shaded: average across plates $(\mathrm{N}=$ 6 per condition), line: smoothed average window $=10 \mathrm{~s})$. 

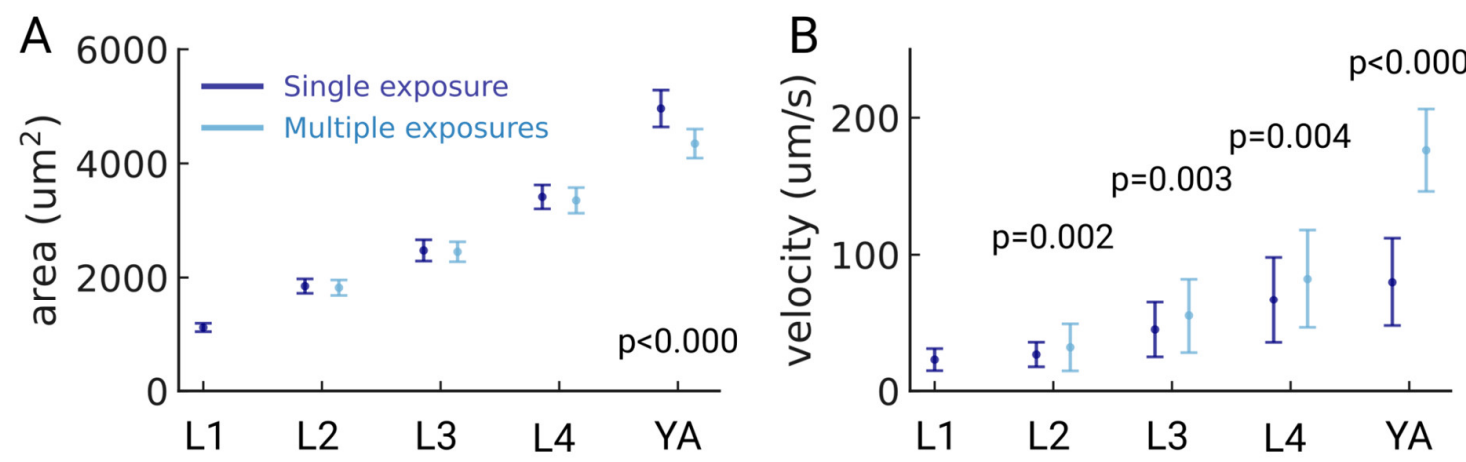

C
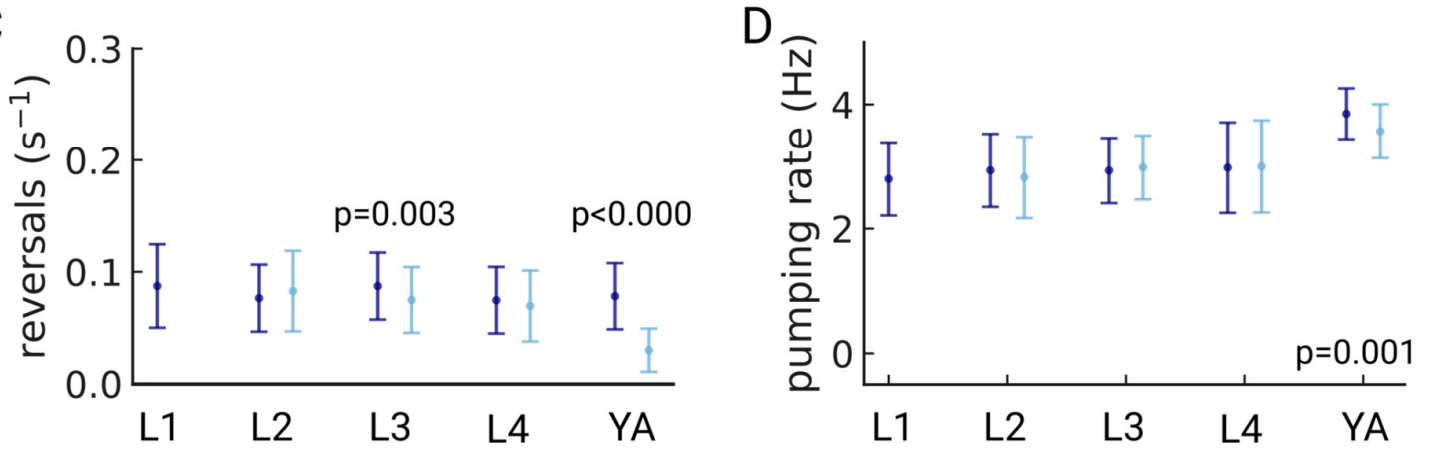

Figure S3.3 Possible light-induced behavioral changes

(A) Pharyngeal area of the detected animals for the group of animals that were imaged once (dark blue) or multiple times (light blue). (B) velocity, (C) reversal rate and (D) pumping rate for the same two cohorts. $p$-values are reported at the bottom for all $p$-values $<0.01$. (Welch's unequal variance twotailed t-test). The sample size of each group was L1 (191/144), L2 (132/153), L3 (87/114), L4 $(112 / 59)$ and YA (38/142), for $\mathrm{N}_{\text {multiple, }} / \mathrm{N}_{\text {Single, }}$ respectively.
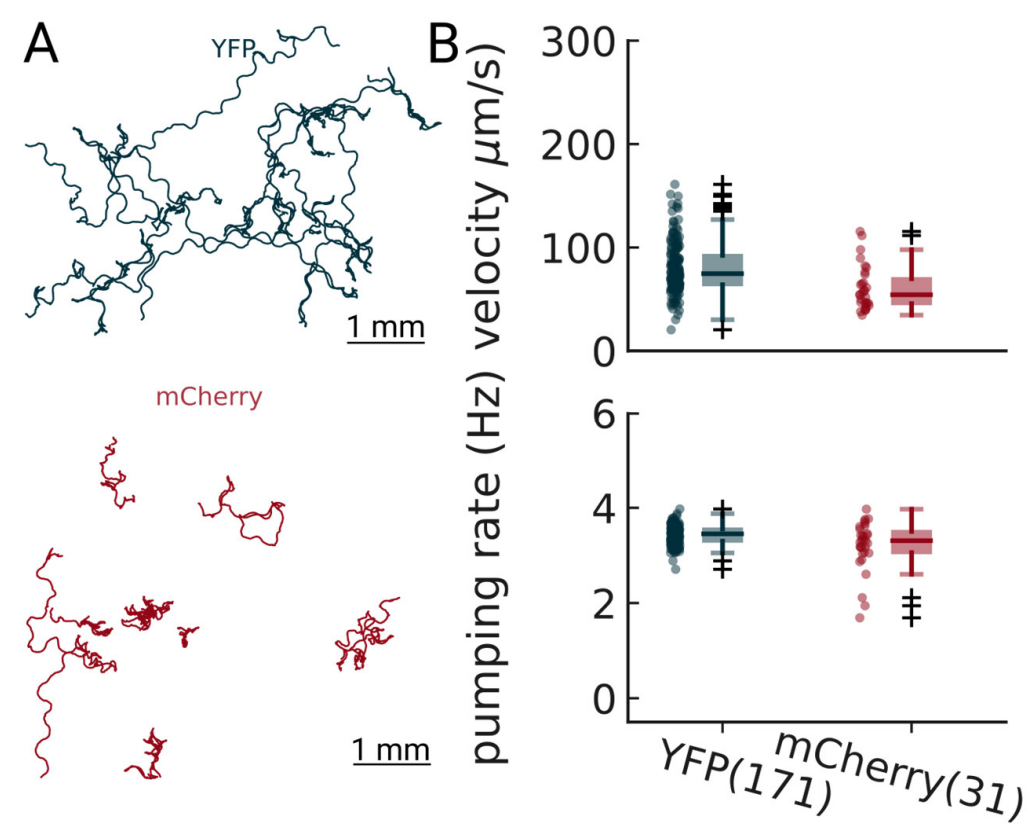

Figure S3.4 Pumping detection is robust at two different excitation wavelengths

Animals expressing either YFP (blue) or mCherry (red) were imaged on food and analyzed using Pharaglow. The pumping rates are not significantly different (Welch's unequal variance two-tailed ttest). 

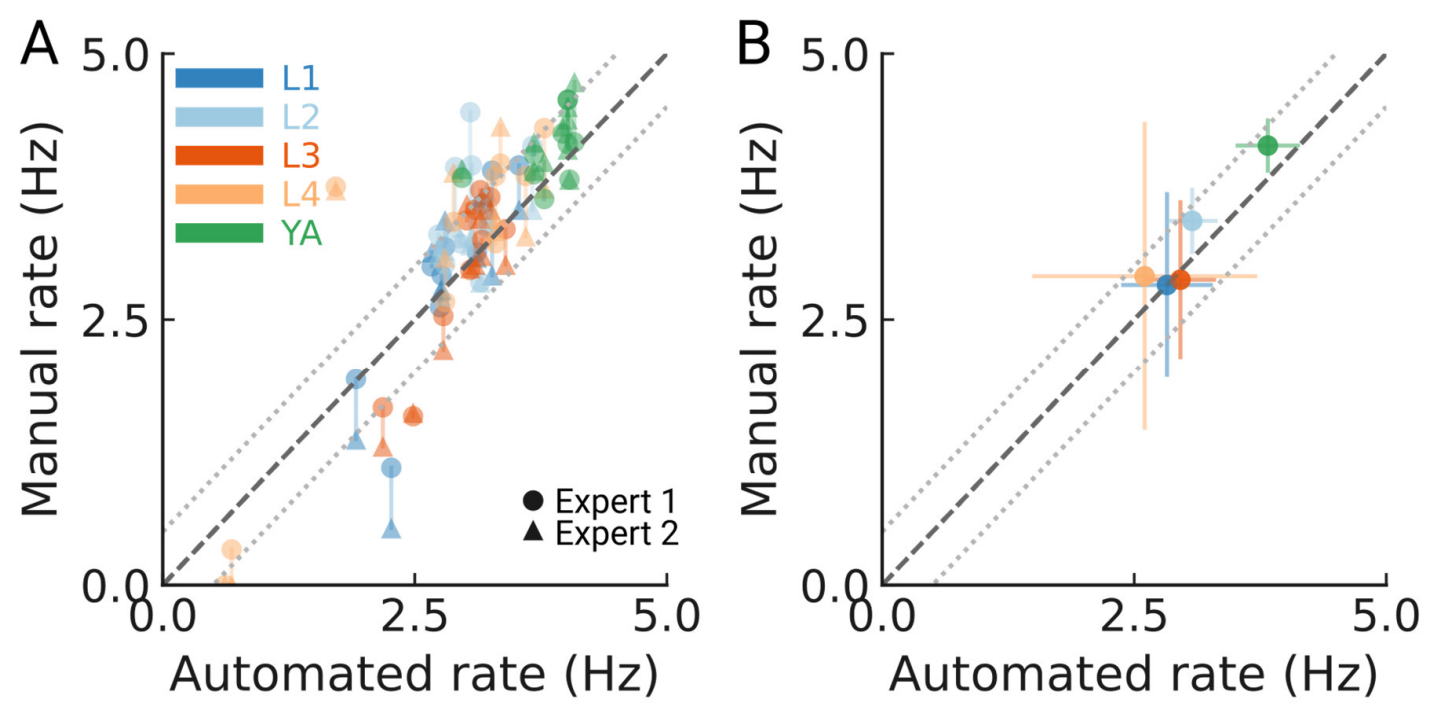

Figure S3.5 Detection accuracy for all developmental stages

(A) Manually counted pumping events for 10 worms per stage (color coded) from two independent experts compared to the automated tracking. Counts of each expert are shown as a circle or triangle. The dashed line indicates unity; the dotted lines denote a $0.5 \mathrm{~Hz}$ difference in the resulting pumping rate. (B) The mean pumping rates per developmental stage from the experts and automated method $(\mathrm{N}=10$ animals per stage). The error bars are s. $d$ between animals. The two expert counts were averaged to obtain one mean count per animal. The dashed line indicates unity; the dotted lines denote a $0.5 \mathrm{~Hz}$ rate difference. 


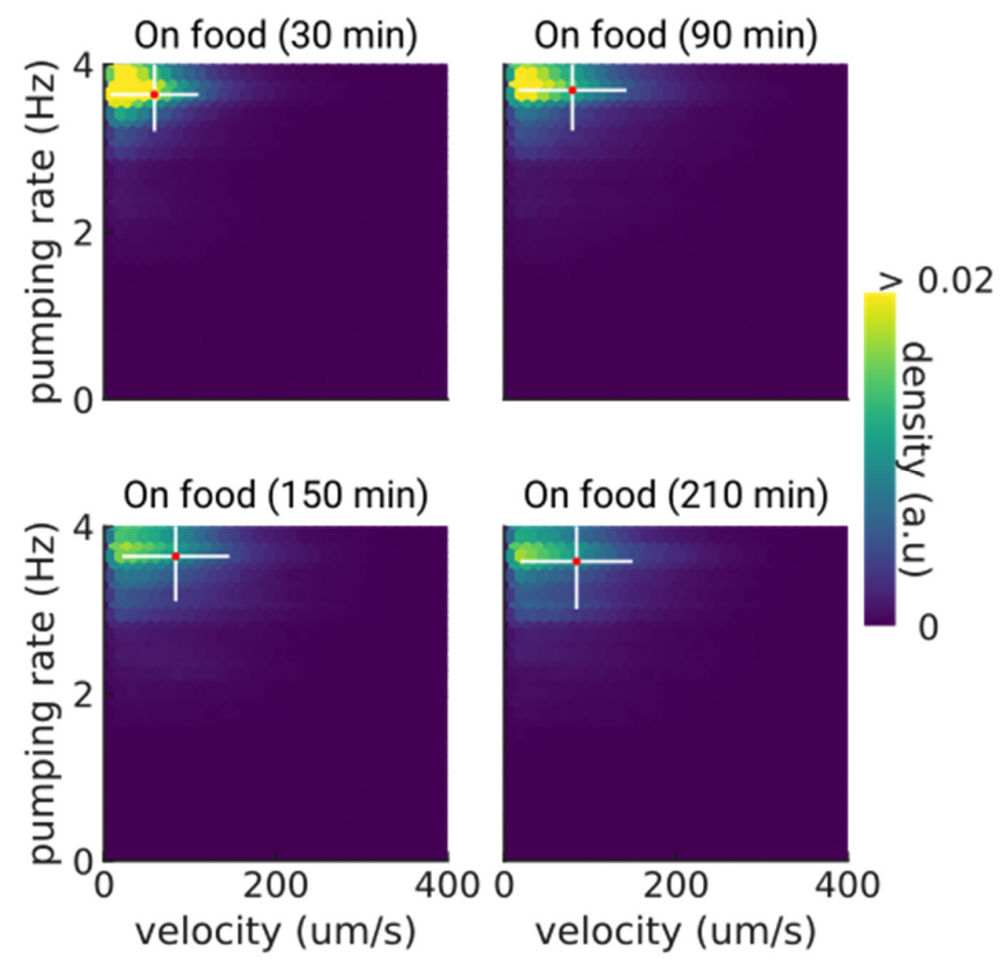

Figure S4.1 Pumping and velocity correlation on food

Correlation between velocity and pumping rate for increasing times on food. The cross indicates the mean (red) and standard deviation (white). The density is normalized by sample number.
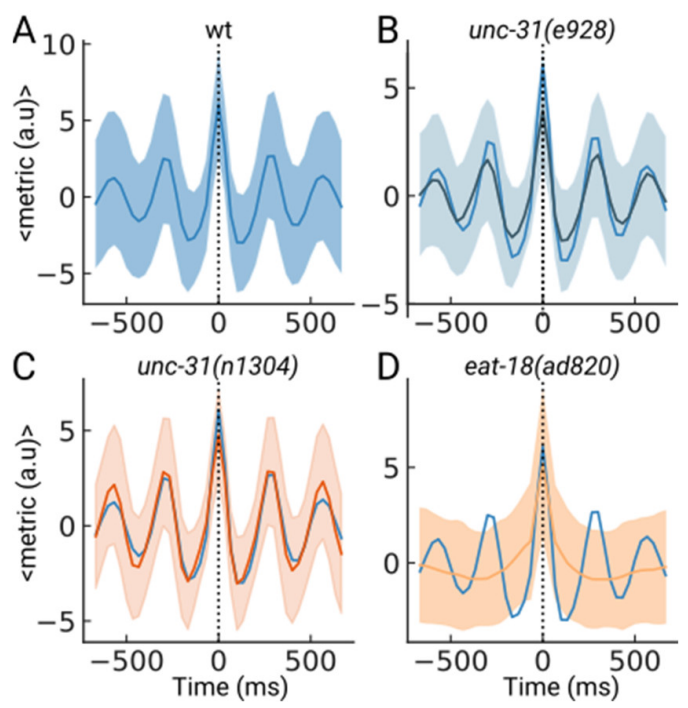

Figure S5.1 Peak-triggered average of the pumping metric.

(A) Average of the pumping metric around the detected peaks of $\mathrm{N}=10$ randomly selected sample animals. The shaded area denotes the standard deviation. (B), (C), (D) same as (A) but the wildtype peak shape is underlaid in dark blue.

\section{Supplementary data}

Video S1 of a GRU101 worm pumping with the pumping metric shown

Video $\mathrm{S} 2$ eat-18 animal pumping 